\title{
Dynamic Decomposition of Poincaré Plots for Multivariate Analysis and Visualization of Simultaneously Recorded Physiological Time Series
}

\author{
J. Piskorski ${ }^{1 *}$, P. Guzik ${ }^{2}$ \\ ${ }^{1}$ Institute of Physics, University of Zielona Góra, ul. Szafrana 4a, Zielona Góra, Poland \\ ${ }^{2}$ Department of Cardiology - Intensive Therapy and Internal Diseases, Poznań University of Medical Sciences, \\ ul. Przybyszewskiego 49, Poznań, Poland \\ *e-mail: J.Piskorski@proton.if.uz.zgora.pl
}

(Received: 23 March 2010; accepted 12 July 2010; published online: 13 September 2010)

\begin{abstract}
We apply the dynamic decomposition of Poincaré plots, which is a computationally intensive, visual method of analysing physiological time series, to the analysis of the interbeat interval variability, systolic blood pressure, stroke volume and total peripheral resistance which were simultaneously recorded from a patient with pheochromocytoma and rapid, repetitive haemodynamic changes over 6 hours. The resulting animation is analysed and interpreted. It is found that changes in total peripheral resistance usually precede those of other variables, and the magnitude of changes is greatest for this variable. It is demonstrated that the decomposition of Poincaré plots of multivariate signals can visualise both the order and the extent of ongoing instant changes.
\end{abstract}

Key words: heart rate variability, Poincaré plot, haemodynamics, pheochromocytoma, medical visualisation

\section{INTRODUCTION}

Heart rate variability is usually studied through the analysis of numerical descriptors such as the spectral power in various frequency bands, descriptors of the Poincaré plot, nonlinear indices related to dynamical systems analysis, such as the correlation dimension, Lyapunov exponents or various kinds of entropy, or indices describing the complexity of strings which are the result of mapping the $R R$-time series onto a string consisting of a finite number of symbols as in the various approaches to symbolic dynamics [1-5].

All the above are not always immediately understandable to a medical expert without an extensive mathematical preparation. However, one of the methods mentioned, namely the Poincaré plot, has also a visual aspect. The Poincaré plot forms a visual summary of the length of cardiac cycles time series derived from an ECG recording (or any other variable), which can be analysed by inspection and interpreted.

The cloud of points which forms the plot may correspond to ECG recordings of various lengths - from $5 \mathrm{mi}$ nutes to 24 hours, and possibly longer. The Poincaré plots constructed from longer recordings are sometimes difficult to interpret. Since there are thousands of points in the plotting area, there is a lot of overlap and the fine aspects of the plot are hidden.

To overcome this difficulty we have devised a computationally intensive, visual method of dynamic decomposition of the Poincare plot, which can summarise both the resulting plot and the process which leads to its creation. In the present paper we apply this method to the multivariate time series of simultaneously recorded haemodynamic variables. Additionaly, the mathematical and algorhythmic aspects of both the univariate and multivariate dynamical Poincaré plot are derived and described for the first time. To demonstrate the utility of the method we apply it to the case of a patient with pheochromocytoma accompanied by repetitive rises and falls of systolic blood pressure (compare the description of case [6]). The following time series are simultaneously analysed: interbeat interval (IbI), systolic blood pressure (SBP), stroke volume (SV) and total peripheral resistance (TPR).

A similar, univariate method was used to demonstrate the process of creation of the complex Poincaré plot shapes 
of RR intervals only [7], the effect of various filters on the creation of Poincaré plots ([8] and the accompanying animations) and to show the stability of the position of the centroid of the Poincare plot with respect to the identity line and its instability with respect to any line perpendicular to the line of identity ([9] and the accompanying animation). However, those papers presented a totally different aspect of the dynamic analysis (multivariate case study in the present paper vs. univariate process analysis in the earlier ones). Also, no mathematical and algorhytmic details were given there.

\section{THE CONSTRUCTION OF THE POINCARÉ PLOT}

The Poincaré plot is constructed from the vector holding the analysed time series

$$
\mathbf{x}=\left(x_{1}, x_{2}, \ldots, x_{N+1}\right),
$$

where $x_{i}$ is the $i$-th element of the time series and $N+1$ is its length.

Let us define two auxiliary vectors:

$$
\begin{aligned}
& \mathbf{x}^{+}=\left(x_{1}, x_{2}, \ldots, x_{N}\right)=\left(x_{1}^{+}, x_{2}^{+}, \ldots x_{N}^{+}\right), \\
& \mathbf{x}^{-}=\left(x_{2}, x_{3}, \ldots, x_{N+1}\right)=\left(x_{1}^{-}, x_{2}^{-}, \ldots x_{N}^{-}\right) .
\end{aligned}
$$

The Poincaré plot is the set of all ordered pairs:

$$
P P=\left\{\left(x_{i}^{+}, x_{i}^{-}\right): x_{i}^{+} \in \mathbf{x}^{+}, x_{i}^{-} \in \mathbf{x}^{-}, i=1, \ldots, N\right\},
$$

where $x_{i}^{+}$and $x_{i}^{-}$are elements of the respective vectors

The basic Poincaré plot descriptors are defined as [2]:

$$
S D 1=\sqrt{\operatorname{Var}\left(\frac{\mathbf{x}^{+}-\mathbf{x}^{-}}{\sqrt{2}}\right)}, \quad S D 2=\sqrt{\operatorname{Var}\left(\frac{\mathbf{x}^{+}+\mathbf{x}^{-}}{\sqrt{2}}\right)} .
$$

Let us define the instantaneous Poincaré plot of length $k$ at position $j\left(P P_{k}^{j}\right)$ as a Poincaré plot (3) based on the following vector:

$$
\mathbf{x}_{k}^{j}=\left(x_{j}, x_{j+1}, \ldots, x_{j+k-1}\right) .
$$

We can also define the instantaneous Poincaré plot of length $T$ at time $t\left(P P_{T}^{t}\right)$ (that is the base our analysis on units of time, rather than beat number), as a Poincaré plot (5) based on the following vector:

$$
\mathbf{x}_{T}^{t}=\left(x_{j}, x_{j+1}, \ldots, x_{j+k-1}\right),
$$

where

$$
j=\max \left\{l: \sum_{i=1}^{l} x_{i} \leq t\right\}, \quad k=\max \left\{l: \sum_{i=j}^{j+l} x_{i} \leq T\right\} .
$$

The other expressions (2, 3 and 4) remain unchanged, but they use $\mathbf{x}_{k}^{j}$ or $\mathbf{x}_{T}^{t}$ rather than $\mathbf{x}$.

Dynamic decomposition of the Poincaré plot of length $k$ (or $T$ ) of a time series consists of a list of all instantaneous Poincaré plots:

$$
\mathbf{P P}_{k}=\left(P P_{k}^{1}, P P_{k}^{2}, \ldots, P P_{k}^{N+1-k}\right),
$$

or, using units of time rather than beats

$$
\begin{aligned}
& \mathbf{P P}_{T}=\left(P P_{T}^{t_{1}}, P P_{T}^{t_{2}}, \ldots, P P_{T}^{t_{M}}\right) \\
& M=\max \left\{l: \sum_{i=1}^{l} x_{i} \leq\left(\left\lfloor t_{\mathrm{tot}} / T\right\rfloor-1\right) \cdot T\right\},
\end{aligned}
$$

where $t_{\text {tot }}$ is the total length of the recording (in units of time), $t_{i}$ is the time of the $i$ th event, corresponding to $x_{i}$, and the symbol $\lfloor x\rfloor$ stands for the integral part of $x$.

If all the instantaneous Poincaré plots are actually drawn and compressed into an animation, we get a tool which lets us analyse the time evolution of the instantaneous Poincaré plot. This method combines the static Poincaré plot method (note that in the definition (3) the ordering of the indices $i$ is irrelevant, as SD1 and SD2 are strictly statistical measures), and the time dependent aspect of time evolution defined by the ordered, increasing indices in (7) or (8). Note also that the calculation of the descriptors, $S D 1$ and $S D 2$, for all the instantaneous Poincaré plots results in a time series which keeps track of the changes of long and short term variability at the preselected beat or time scale $k$ or $T$ (compare (5), (6) and Section III).

\section{INTERPRETATION OF THE POINCARÉ PLOT AND ITS DESCRIPTORS}

An example Poincaré plot is presented in Fig. 1. The reference line for all the points of the Poincare plot is the line of identity. Above this line, the elements of the Poincaré plot (3) have the following property: $\left(x_{i}^{+}<x_{i}^{-}\right)$ or, using the original vector (1) $\left(x_{i}<x_{i+1}\right)$, below this line we have $\left(x_{i}^{+}>x_{i}^{-}\right)$or $\left(x_{i}>x_{i+1}\right)$, and on the line of identity $\left(x_{i}^{+}=x_{i}^{-}\right)$, or $\left(x_{i}=x_{i+1}\right)$. If we focus our attention on a specific time series, say, the interbeat interval series (IbI), we can conclude that all the prolongations of the IbI are depicted above the identity line, all shortenings are below this line, and the points on the line correspond to two equal consecutive IbIs [8].

The descriptors $S D 1$ and $S D 2$ defined by (4) measure the dispersion of points belonging to the Poincare plot $S D 1$ measures the dispersion across the line of identity and $S D 2$ measures dispersion along this line. It is believed that 
$S D 1$ corresponds to short-term variability, and $S D 2$ to long-term variability [2].

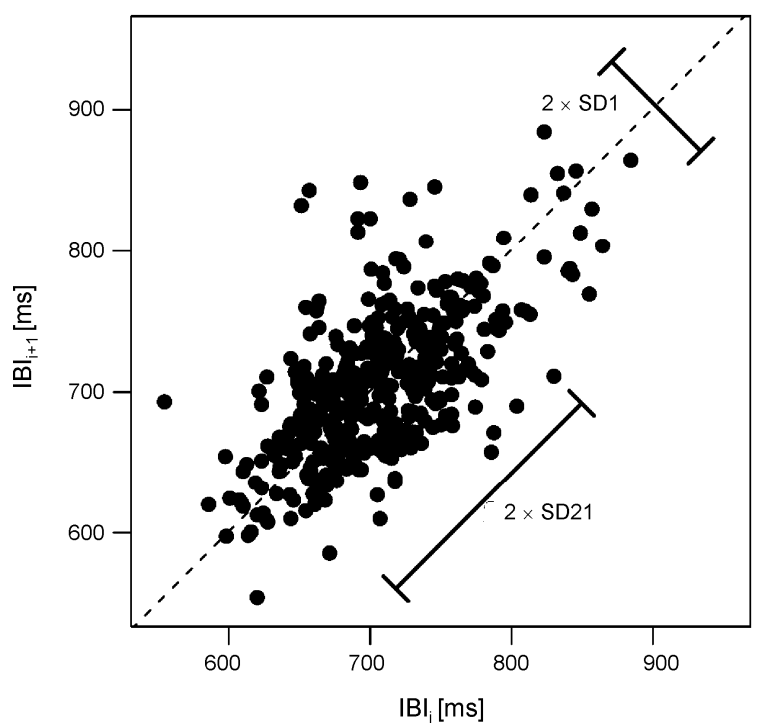

Fig. 1. An example Poincaré plot of interbeat intervals from a 5 min recording of a healthy person with the basic descriptors and the line of identity (the dashed line)

\section{MATERIALS AND METHODS}

For the decomposition of the Poincaré plot of multivariate cardiovascular time series we used data from a 52-year-old woman with pheochromocytoma, i.e. a neuroendocrine tumor usually localised in the medulla of the adrenal glands. Pheochromocytoma cells can secrete excessive amounts of catecholamines, usually epinephrine and norepinephrine, causing dramatic cardiovascular oscillations. The patient experienced repetitive blood pressure changes which were accompanied by symptoms of hypertensive crisis (SBP $>300 \mathrm{mmHg}$ ) or cardiogenic shock (SBP $<50 \mathrm{mmHg}$ ) [9]. Beat-to-beat finger blood pressure waveform was recorded with Portapres-2 (FMS, the Netherlands) for 346 minutes. SBP and IbI were obtained from the original pulse pressure curve whereas the values of SV and TPR were reconstructed with the use of the Modelflow algorithm [10].

To avoid the inclusion of potential artifacts, premature beats and post-ectopic compensatory pauses, we applied filters based on the IbI time series removing haemodynamics for the following types of beats:

- $\mathrm{IbI}<300 \mathrm{~ms}$ and $\mathrm{IbI}>2000 \mathrm{~ms}$,

- $\mathrm{IbI}_{n} / \mathrm{IbI}_{n+1}>0.3, \mathrm{IbI}_{n+1} / \mathrm{IbI}_{n}>0.3$.
Corresponding data points from the other three time series were also removed with the use of the methods described in [8].

The software for Poincaré plot decomposition was written in Matlab (MathWorks, USA). Each frame of the animation was exported to disc as a png file and then all frames were compressed into a single file with the use of the Linux application mencoder (the result in various formats may be downloaded from [11]).

\section{ANIMATION}

We use an 800-beat sliding window ${ }^{1}$ along the IbI-, SBP-, TPR- and SV-grams (see Fig. 2). The instantaneous Poincaré plot $s\left(P P_{800}^{k}\right)$ for each segment with values of $S D$, $S D 1$ and $S D 2$ for all normalised variables (i.e. divided by the respective means) are shown on the right.

The variables used in Poincaré plot decomposition were normalised by their respective total (from the whole recording) means and presented in relative units to make it possible to compare the variability of the component signals between one another. The IbI-, SBP-, TPR- and SVgrams are presented in the original units.

The simultaneous analysis reveals that the alterations of TPR usually lead the remaining haemodynamic changes, with the slowest and most delayed response in SV. The highest range of changes is found in TPR and the smallest in IbI. The shapes of the plots are different for all haemodynamic time series: they are cigar-like for SBP and TPR, more elliptic for IbI and the widest for SV.

Visual inspection of the recorded and decomposed cardiovascular signals (Fig. 2 and the animations [11]) helps understand clinical manifestations of the disease. Pheochromocytoma secretes various substances, including catecholamines and their metabolites which act on the cardiovascular system $[6,12]$. In case of the described patient, the tumor was periodically releasing large amounts of norepinephrine and epinephrine (blood concentrations of both hormones were way out of normal range, i.e. norepinephrine was $7924 \mathrm{pg} / \mathrm{mL}$ (upper normal limit to $374 \mathrm{pg} / \mathrm{mL}$ ) and epinephrine was $85.4 \mathrm{pg} / \mathrm{mL}$ (upper normal limit of $48 \mathrm{pg} / \mathrm{mL}$ ). Hypersecretion of these catecholamines is responsible for clinical manifestations of pheochromocytoma like episodic headache, sweating,

\footnotetext{
1 The selection of the length of the window ( 800 beats) deserves some explanation. We noticed by experimenting with various lengths that if more beats are selected many fine details of the animation are lost. On the other hand, if the window is shorter, the plot becomes a collection of separate points rather than a well-defined shape. We do not believe that a single length can be established for every recording; rather, it is necessary to adjust it to the specific case under consideration.
} 

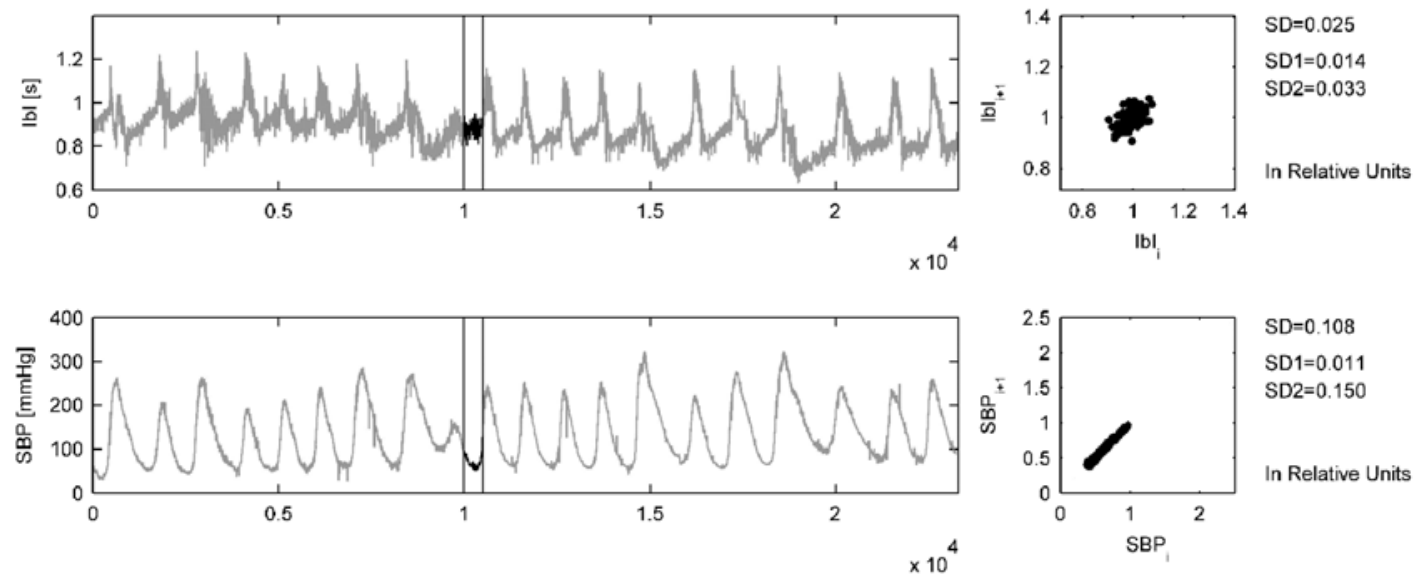

$\mathrm{SD}=0.108$

$\mathrm{SD} 1=0.011$

$\mathrm{SD} 2=0.150$
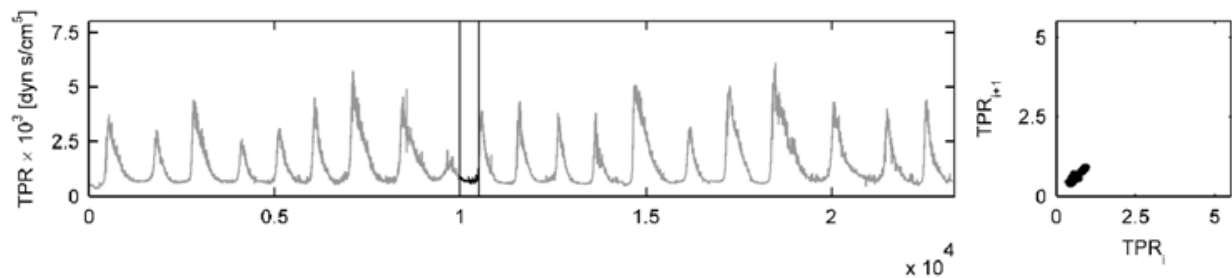

$\mathrm{SD}=0.066$

$\mathrm{SD} 1=0.018$

SD2 $=0.090$

In Relative Units
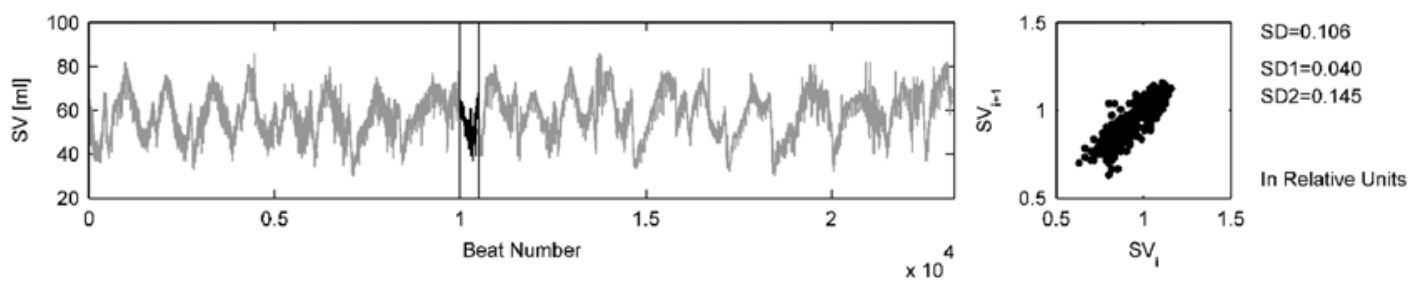

Fig. 2. A frame from the dynamic decomposition of a 6-hour multivariate recording of interbeat interval (IbI), systolic blood pressure (SBP), stroke volume (SV) and total peripheral resistance (TPR). An 800-beat sliding window moves along the IbI-, SBP-, TPR- and SV-grams. The corresponding Poincaré plots (right side) show the variation in relative units (variables divided by their respective means) and are accompanied by numerical descriptors. For details see main text. The animation can be downloaded from [11]

tachycardia and rapid increase or decrease of blood pressure leading to hypotension or hypertension, respectively. The haemodynamic fluctuations are visible both in the animation and Fig. 2. In our patient the cyclic nature of changes of heart rate (the IBI panel, which is the reverse of heart rate), blood pressure (the SBP panel), vascular resistance (the TPR panel) and the amount of blood ejected from the left ventricle of the heart (the SV panel) can be easily found every 14 to 21 minutes. During this time, patient presented with paroxysmal attacks of headache, strong epigastric pain, nausea, agitation and anxiety or weakening, sleepiness and blurred vision, her skin was wet, cold and pale or it was dry, warm and red. All of the symptoms might be explained by changes of vascular resistance and tissue blood perfusion which are secondary to alterations of TPR, SBP, IBI and SV.

The total variability of the analyzed data (SD) of each 800-beat window is the highest for TPR and SBP, then SV and the smallest for IBI. Depending on the time of the recording, SD1 and SD2 change rapidly, but on the average their alterations are the biggest for TPR, then for SBP, SV and IBI. The analysis of the sequence of changes in the IBI-, SBP-, TPR- and SV-grams and their mini-Poincaré plots reveals that TPR alterations precede changes in IBI, then SBP with the most delayed response in SV. Under physiological conditions, changes in IBI, called heart rate variability, buffer the changes in blood pressure to secure a relatively constant blood perfusion to tissues and organs $[13,14]$. This is why the relative changes of IBI are usually bigger than those of SBP. Although the TPR changes are the earliest in the patient discussed in the present paper, they do not buffer other haemodynamic changes leading to their increased variability and rapid oscillations in blood perfusion. It is probable that catecholamines released by the tumor cells in an oscillatory pattern are responsible for the dramatic and strong changes in vascular tone (repre- 
sented here by TPR) and autonomic activity leading to reflex alterations in SBP, IBI and SV. The observed episodes of vasoconstriction/vasodilation override the physiological compensatory mechanisms and force a new sequence of haemodynamic changes. As can be seen, the method offers a wealth of physiological information which might otherwise be difficult to obtain. If one was to analyze the simultaneous recordings by the signal-analytic numerical methods, one would be forced to use complex multivariate methods and would have to be able to interpret the numerical results. In the described method it is the human eye that recognizes patterns and no expert knowledge of signal analysis is required. The more advanced numerical analysis might follow the visual inspection and initial interpretation.

\section{CONCLUSION}

We have described a new visual method for simultaneous analysis of multivariate physiological time series: the decomposition of Poincaré plots. The method has been carefully described mathematically so that it can be reproduced without any ambiguities.

The decomposition has been applied to the analysis of the multivariate signal of heart rate, blood pressure, stroke volume and total peripheral resistance recorded from a patient with pheochromocytoma accompanied by repetitive rises and falls of systolic blood pressure. It has been found that in this patient the TPR changes were the earliest and their range was the biggest.

As shown in the paper, the method can be applied to any cardiovascular time series recorded simultaneously and representing different haemodynamic parameters. The decomposition of Poincaré plots summarizes the whole set of component time series of a multivariate time series by showing how local variability changes in time. Some other areas of potential application include sports medicine, analysis of recordings acquired during patient monitoring, analysis of data from the monitoring of persons working in extreme or unusual conditions like aircraft pilots, divers or even astronauts and soldiers on a battlefield and to study and better understand mutual relationship between various signals like blood pressure and heart rate as well as visualize the magnitude of changes of the analyzed signals. In general, the method could be useful in any analysis where global variability may be understood as a net effect of well-defined local changes.

\section{References}

[1] A.J.E. Seely, P.T. Macklem, Complex systems and the technology of variability analysis. Critic. Care 6, R367-84 (2004).

[2] M. Brennan, M. Palaniswami, P. Kamen, Do existing measures of Poincaré plot geometry reflect nonlinear features of Heart Rate Variability? IEEE Trans. Biomed. Eng. 48, 1342-1347 (2001).

[3] A. Voss, R. Schroeder, G. Trubner, M. Goering, H.R. Figulla, A. Schirdewan, Comparison of nonlinear methods symbolic dynamics, detrended fluctuation, and Poincaré plot analysis in risk stratification in patients with dilated cardiomyopathy. Chaos 17, 015120-1-7 (2007).

[4] S. Guzzetti, M.G. Signorini, C. Cogliati, S. Mezzetti, A. Porta, S. Cerutti, A. Malliani, Non-linear dynamics and chaotic indices in heart rate variability of normal subjects and heart-transplanted patients. Cardiovasc. Res. 31, 441-446 (1995).

[5] M. Costa, A.L. Goldberger, C K. Peng, Multiscale entropy analysis of biological signals. Phys. Rev. E 71, 021906-118 (2005).

[6] P. Guzik, A. Wykretowicz, K.H. Wesseling, H. Wysocki, Adrenal pheochromocytoma associated with dramatic cyclic haemodynamic fluctuations. Int. J. Cardiol. 103, 351-353 (2005).

[7] P. Guzik, J. Piskorski, Decomposition of the commet-like Poincaré plot $s$ of RR intervals. Folia Cardiol. 12 suppl. D, O271-4 (2005).

[8] J. Piskorski, P. Guzik, Filtering Poincaré plots, Comp. Meth. Sci. Tech. 11, 39-48 (2005)

[9] J. Piskorski, P. Guzik, Geometry of the Poincaré plot and its asymmetry in healthy adults. Phys. Meas. 28, 287-300 (2007).

[10] J.J. van Lieshout, K. Toska, E.J. van Lieshout, M. Eriksen, L. Walloe, K.H. Wesseling, Beat-to-beat noninvasive stroke volume from arterial pressure and Doppler ultrasound. Eur. J. Appl. Physiol. 90, 131-137 (2003).

[11] The animation described in the present paper can be downladed in various formats from www.if.uz.zgora.pl/ jaropis/dyndesig.html

[12] N.M. Kaplan, Hypertension and atherosclerotic cardiovascular disease. In: Braunwald E, Zipes DP, Libby P. editors. Heart Disease. A Textbook of cardiovascular medicine, 6th Edition, p. 941-971. W.B. Saunders Company (2001).

[13] Task Force of the European Society of Cardiology and the North American Society of Pacing and Electrophysiology, Heart rate variability. Standards of measurement, physiological interpretation, and clinical use. Eur. Heart J. 17, 354-381 (1996).

[14] R.E. Kleiger, P.K. Stein, J.T. Bigger Jr, Heart rate variability: measurement and clinical utility. Ann. Noninvasive Electrocardiol. 10, 88-101 (2005). 


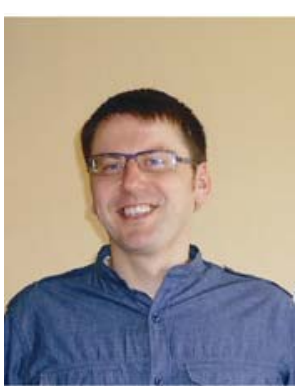

Dr. Jarostaw Piskorski works at the Institute of Physics of the University of Zielona Góra, Poland. His main areas of interest include the application of the methods of statistical physics and nonlinear dynamics to heart rate variability and elementary particle physics (mainly discrete symmetries and their violation). Together with prof. Przemyslaw Guzik, he is co-discoverer of the Heart Rate Asymmetry phenomenon and co-creator of the monotonic runs method in mortality prediction.

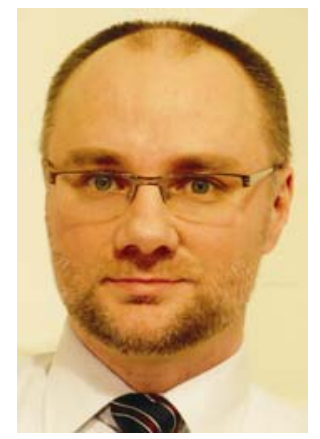

Assoc. Prof. Przemyslaw Guzik is both a medical doctor and scientist working at the Department of Cardiology - Intensive Therapy, University of Medical Sciences in Poznań, Poland. Non-invasive evaluation of cardiovascular system and autonomic modulation of heart rate belong to his research topics. The analysis of various cardiovascular time series (heart rate, blood pressure) allows him to focus on heart rate variability, heart rate turbulence, baroreflex sensitivity and arterial stiffness. The use of Poincare plots of heart rate, which is his principal area of interest and research, is one of the approaches used to investigate heart rate variability. Together with dr. Jaroslaw Piskorski, he is co-discoverer of the Heart Rate Asymmetry phenomenon and co-creator of the monotonic runs method in mortality prediction. 